\title{
Constitutive Equations and Numerical Modelling of Time Effects in Soft Porous Rocks
}

\author{
M. Datcheva ${ }^{1}$, R. Charlier ${ }^{2}$, and F. Collin ${ }^{2}$ \\ ${ }^{1}$ Institute of Mechanics, Bulgarian Academy of Sciences, Sofia \\ ${ }^{2}$ University of Liege, Liege, Belgium
}

\begin{abstract}
A constitutive model is developed within the framework of Perzyna's viscoplasticity for predicting the stress-strain-time behaviour of soft porous rocks. The model is based on the hyperelasticity and multisurface viscoplasticity with hardening. A time-stepping algorithm is presented for integrating the creep sensitive law. An example of application to one-dimensional consolidation is presented. The objectives are to: 1. present a soft rock model which is capable of taking into account the rate sensitivity, time effects and creep rupture; 2 . to discuss the use of an incremental procedure for time stepping using large time increments and 3. to extend the finite element code Lagamine (MSM-ULg) for viscoplastic problems in geomechanics.
\end{abstract}

\section{Introduction}

For solving geomechanical problems, such as well-bore stability, subsidence, hydraulic fracturing and ect., the most important is to deal with a proper constitutive model for the complex soft porous rock mechanical behaviour. Various models have been developed for the time independent behaviour of chalk as a typical soft porous rock. However the failure of an underground structure in this rock may occur due to creep deformation and therefore the use of conventional time-independent procedure for the interpretation of laboratory results and the analysis of geotechnical boundary-valued problems may result in solutions which do not properly capture the actual in situ response. The model proposed in this study is a time-dependent inelastic model for rocks and soils based on the Perzyna's elasto-viscoplastic theory, [6]. Motivations for adopting Perzyna's elastic-viscoplastic theory are:

1. The formulation is well accepted and well used;

2. The generality of the time-rate flow rule offers the capability of simulating time-dependent material behaviour over a wide range of loading;

3. The incorporation of the inviscid multisurface cap-failure-tension model, developed in DIG-ULg in the frame of PASACHALK project, was of interest ([1] and [2]); and

4. The formulation is readily adaptable to a numerical algorithm suitable for finite element procedure and particularly for implementation in Lagamine (MSM-ULg) finite element code.

L. Vulkov, J. Waśniewski, and P. Yalamov (Eds.): NAA 2000, LNCS 1988, pp. 222-229, 2001.

(C) Springer-Verlag Berlin Heidelberg 2001 


\section{Mechanical Model. Perzyna's Viscoplasticity}

Perzyna's theory, [6] is a modification of classical plasticity wherein viscous-like behaviour is introduced by a time-rate flow rule employing a plasticity yield function. The strain rate tensor $\dot{\varepsilon}_{i j}$ is composed of elastic $\dot{\varepsilon}_{i j}^{e}$ and viscoplastic $\dot{\varepsilon}_{i j}^{v p}$ strains, or by the definition:

$$
\dot{\varepsilon}_{i j}=\dot{\varepsilon}_{i j}^{e}+\dot{\varepsilon}_{i j}^{v p} .
$$

The stress rate tensor $\dot{\sigma}_{i j}$ is related to the elastic strain rate via a linear elastic or hyperelastic constitutive tensor $C_{i j k l}$. Therefore, taking into account the relation (1) the elastoplastic constitutive relation between stress and strain rates reads:

$$
\tilde{\sigma}_{i j}=C_{i j k l}\left(\dot{\varepsilon}_{k l}-\dot{\varepsilon}_{i j}^{v p}\right),
$$

The Jaumann type objective time derivative of stress tensor is defined by $\tilde{\sigma}_{i j}=$ $\dot{\sigma}_{i j}+\omega_{i k} \sigma_{k j}+\sigma_{i k} \omega_{k j}^{T}$ where $\boldsymbol{\omega}$ is the anti-symmetric part of the velocity gradient. The viscoplastic flow rule is expressed as:

$$
\dot{\varepsilon}_{i j}^{v p}=\gamma\langle\Phi(f)\rangle \frac{\partial g}{\partial \sigma_{i j}}, \quad\langle\Phi(f)\rangle= \begin{cases}\Phi(f), & f>0 \\ 0, & f \leq 0\end{cases}
$$

in which $\gamma$ is a fluidity parameter; $\Phi$ - viscous flow function; $g=g(\sigma, \varpi)$ - creep potential and $f=f(\sigma, \varpi)$ is any valid plasticity function, playing the role of loading surface. The parameter $\varpi$ stays for some hardening function of the viscoplastic strain history, i.e., $\varpi=\varpi\left(\bar{\varepsilon}^{v p}\right)$, where $\bar{\varepsilon}^{v p}$ is an equivalent viscoplastic strain representing the magnitude of the viscoplastic deformation. For a given value of $\varpi$, all states of stress that satisfy $f=0$ form the current "static" yield surface. The "static" yield surface forms a boundary between elastic $(f \leq 0)$ and viscoplastic $(f>0)$ domains. When a constant stress state is imposed such that $f>0$, viscoplastic flow will occur. If $f$ is a nonhardening yield function the flow will continue to occur at a constant rate. If $f$ is a hardening function, viscoplastic flow occurs at a decreasing rate because as viscoplastic strain accumulates, $\varpi\left(\bar{\varepsilon}^{v p}\right)$ changes in value such that $f(\boldsymbol{\sigma}, \varpi) \rightarrow 0$ and thus $\dot{\varepsilon}_{i j}^{v p} \rightarrow 0$. In this way the static yield surface is moving out on a real time to eventually form a new static yield surface containing the imposed stress state. Once the new static yield surface has stabilized, the steady state solution $\dot{\varepsilon}_{i j}^{v p}=0$ is achieved. The resulting strains accumulated during this loading would be identical to the corresponding time-independent plastic solution.

\subsection{Application to the Soft Porous Rocks}

The concept of two inelastic deformation mechanisms - collapse (volumetric or cap) and shear failure or deviatoric - has been applied to the viscoplastic analysis. Such a concept is based on the experimental observation for high porous rocks and for chalk especially (see [8]). Therefore:

$$
\dot{\varepsilon}_{i j}^{v p}=\dot{\varepsilon}_{i j}^{c}+\dot{\varepsilon}_{i j}^{d},
$$


Indexes $c$ and $d$ are for collapse (cap or volumetric) and deviatoric or shear failure viscoplastic strain components and model parameters respectively. In the present work the loading surface and creep potential are functions of the first, second and third stress invariants: $I_{\sigma}=\sigma_{i j} \delta_{i j}, I I_{s}=\sqrt{\frac{1}{2} s_{i j} s_{i j}}$ and $\beta=-\frac{1}{3} \sin ^{-1}\left(\frac{3 \sqrt{3}}{2} \frac{I I I_{s}}{I I_{s}^{3}}\right)$, where $I I I_{s}=\frac{1}{3} s_{i k} s_{k j} s_{j i}$ and $s_{i j}=\sigma_{i j}-I_{\sigma} / 3$ is the stress deviator. The static yield surface $f=0$ and the creep potential function $g=0$ are divided into two regions along the first stress invariant axis $I_{\sigma}$ : the cap surface region $\left(I_{\sigma}<L=\frac{1}{2}\left(\frac{3 c}{\tan \phi_{C}}-3 p_{0}\right)\right)$ with $f_{c}$ and $g_{c}$ and the failure surface region $\left(I_{\sigma} \geq L\right)$ with $f_{d}$ and $g_{d}$. Here $c$ is the cohesion, $\phi_{C}$ is the friction angle in the compression path and $p_{0}$ is the preconsolidation pressure. Such an approach overcomes difficulties, mentioned in [7] to extend the Perzyna's type viscoplasticity in the case of multisurfase inelasticity.

Cap loading surface $f_{c}$ is a hardening surface defined by

$$
f_{c}=I I_{s}^{2}+m^{2}\left(I_{\sigma}-\frac{3 c}{\tan \phi_{C}}\right)\left(I_{\sigma}+3 p_{0}\right)=0,
$$

where

$$
\begin{aligned}
m & =a(1+b \sin 3 \beta)^{n}, \\
b & =\frac{\left[\sin \phi_{C}\left(3+\sin \phi_{E}\right)\right]^{\frac{1}{n}}-\left[\sin \phi_{E}\left(3-\sin \phi_{C}\right)\right]^{\frac{1}{n}}}{\left[\sin \phi_{C}\left(3+\sin \phi_{E}\right)\right]^{\frac{1}{n}}+\left[\sin \phi_{E}\left(3-\sin \phi_{C}\right)\right]^{\frac{1}{n}}}, \\
a & =\frac{1}{\sqrt{3}} \frac{2 \sin \phi_{C}}{3-\sin \phi_{C}}(1+b)^{-n},
\end{aligned}
$$

$n$ is a model parameter and $\phi_{E}$ is the friction angle in the extension stress path. We assume that:

Hypothesis 1 Hardening for the cap surface is due to the volumetric inelastic strain - $\varepsilon_{v}^{c}$ and therefore $f_{c}=f_{c}\left(\boldsymbol{\sigma}, \varepsilon_{v}^{c}\right)$. The only hardening variable for the cap surface, $p_{0}$, depends only on $\varepsilon_{v}^{c}$.

The hardening law is given as:

$$
\dot{p}_{0}=\frac{1+e}{\lambda-\kappa} p_{0} \dot{\varepsilon}_{v}^{c}
$$

with $e$ - the void ratio, $\lambda$ - the slope of the virgin consolidation line and $\kappa$ - the slope of swell/recompression line in $e-\ln \left(I_{\sigma} / 3\right)$ space. For the cap deformation mechanism $f_{c} \equiv g_{c}$ and referring to the eq. (3), the associated viscoplastic law is:

where

$$
\dot{\varepsilon}_{i j}^{c}=\gamma_{c}\left\langle\Phi_{c}\left(f_{c}\right)\right\rangle \frac{\partial f_{c}}{\partial \sigma_{i j}}
$$

$$
\Phi_{c}=\left(\frac{f_{c}}{p_{0}}\right)^{\alpha_{c}} \text { and } \quad \gamma_{c}=\omega\left(\frac{\left|I_{\sigma}\right|}{3 p_{a}}\right)^{\iota}
$$

with $\omega, \iota$ and $p_{a}-$ material constants. 
Failure loading surface is a hardening, Van Eekelen [4] yield function:

$$
f_{d}=I I_{s}+m\left(I_{\sigma}-\frac{3 c}{\tan \phi_{C}}\right)=0 .
$$

Hypothesis 2 For the failure surface there is no hardening due to the cap type deformation. The equivalent deviatoric inelastic strain

$$
\bar{e}_{d}=\int_{0}^{t} \sqrt{\left(\dot{\varepsilon}_{i j}^{d}-\frac{1}{3} \dot{\varepsilon}_{k l}^{d} \delta_{k l} \delta_{i j}\right)\left(\dot{\varepsilon}_{i j}^{d}-\frac{1}{3} \dot{\varepsilon}_{k l}^{d} \delta_{k l} \delta_{i j}\right)} d \tau
$$

is the only hardening parameter for the failure surface, so $f_{d}=f_{d}\left(\boldsymbol{\sigma}, \bar{e}_{d}\right)$ and thus the internal state variables for the failure deformation mechanism - the friction angles and the cohesion - are functions only of $\bar{e}_{d}$.

For the internal state variables the concrete expressions, explored in the present work are given like in [3]:

$$
\begin{aligned}
\phi_{C} & =\phi_{C 0}+\left(\phi_{C f}-\phi_{C 0}\right) \frac{\bar{e}_{d}}{B_{p}+\bar{e}_{d}}, \\
\phi_{E} & =\phi_{E 0}+\left(\phi_{E f}-\phi_{E 0}\right) \frac{\bar{e}_{d}}{B_{p}+\bar{e}_{d}}, \\
c & =c_{0}+\left(c_{f}-c_{0}\right) \frac{\bar{e}_{d}}{B_{c}+\bar{e}_{d}},
\end{aligned}
$$

where $\phi_{C 0}, \phi_{E 0}, c_{0}$ and $\phi_{E f}, \phi_{E f}, c_{f}$ are initial and final friction angles and cohesion in compression $(C)$ and extension $(E)$ stress paths. Coefficients $B_{p}$ and $B_{c}$ have the values of the equivalent deviatoric inelastic strain for which half of the hardening on friction angles and cohesion is achieved, see [3].

The viscoplastic flow law is non-associated and taking into account (3) it is given by:

$$
\dot{\varepsilon}_{i j}^{d}=\gamma_{d}\left\langle\Phi_{d}\left(f_{d}\right)\right\rangle \frac{\partial g_{d}}{\partial \sigma_{i j}}
$$

with $\Phi_{d}=\left(\frac{f_{d}}{p_{0}}\right)^{\alpha_{d}}, \quad \gamma_{d}=\gamma_{c} a_{2}$, where $\alpha_{d}$ and $a_{2}$ are material constants.

The potential function $g_{d}$ depends on the dilatancy angles $\psi_{C}$ and $\psi_{E}$ in compression and extension paths respectively, and is given as:

$$
g_{d}=I I_{s}+m^{\prime}\left(I_{\sigma}-\frac{3 c}{\tan \phi_{C}}\right)
$$

where

$$
\begin{aligned}
m^{\prime} & =a^{\prime}\left(1+b^{\prime} \sin 3 \beta\right)^{n} \\
b^{\prime} & =\frac{\left[\sin \psi_{C}\left(3+\sin \psi_{E}\right)\right]^{\frac{1}{n}}-\left[\sin \psi_{E}\left(3-\sin \psi_{C}\right)\right]^{\frac{1}{n}}}{\left[\sin \psi_{C}\left(3+\sin \psi_{E}\right)\right]^{\frac{1}{n}}+\left[\sin \psi_{E}\left(3-\sin \psi_{C}\right)\right]^{\frac{1}{n}}} \\
a^{\prime} & =\frac{1}{\sqrt{3}} \frac{2 \sin \psi_{C}}{3-\sin \psi_{C}}\left(1+b^{\prime}\right)^{-n}
\end{aligned}
$$


Here the well known Taylor rule: $\phi_{C}-\psi_{C}=\phi_{E}-\psi_{E}=$ const is used, which is based on experimental evidences.

\section{Numerical Algorithm}

This section concerns a way for implementing the Perzyna's type viscoplasticity in the finite element code for large deformation inelastisic analysis Lagamine, [5]. It is presumed that the strain history is specified and the objectives is to determine the corresponding stress history. Using a step-by-step time integration scheme a numerical solution algorithm is developed at the constitutive level. The time increments realized are large and the nonlinearity is much higher than for the classical elastoplastic laws. The errors that are introduced by the integration scheme can be significantly reduced by sub-incrementation. In the code Lagamine the time increment $\Delta t=t^{B}-t^{A}$, where $B$ indicates the end of the time step and $A$ its beginning, is divided into a constant number $N$ of subintervals with a length $\Delta_{N} t$. For each sub-interval we have to integrate the eq. (3), which an incremental form is:

$$
\Delta \boldsymbol{\sigma}=\boldsymbol{C}(\boldsymbol{\sigma})\left(\Delta \varepsilon-\Delta \varepsilon^{v p}\right)
$$

The right side of (20) depends on $\sigma$ and the hardening function $\varpi$. The problem posed is therefore to know which stress state and value of $\varpi$ to introduce in the right side of (20). A generalised mid-point algorithm is adopted here, where the viscoplastic strain increment is approximated by one-parameter time integration scheme as:

$$
\begin{gathered}
\Delta \varepsilon^{v p}=\Delta_{N} t\left[\left.(1-\theta) \dot{\varepsilon}^{v p}\right|_{A}+\left.\theta \dot{\varepsilon}^{v p}\right|_{B}\right], \\
0 \leq \theta \leq 1 .
\end{gathered}
$$

In each Gauss integration point the following operations would be carried:

1. Use the stress rate from the previous sub-interval $i-1$ and the stress $\boldsymbol{\sigma}^{A}$ at the beginning of the step $\Delta t$ to evaluate a mid-point stress $\sigma^{\theta}$ :

$$
\boldsymbol{\sigma}^{\theta}=\boldsymbol{\sigma}^{A}+\tilde{\boldsymbol{\sigma}}_{i-1} \theta \Delta_{N} t
$$

Hardening parameters, $\varepsilon_{v}^{c}$ for the cap and $\bar{e}_{d}$ for the failure regions are evaluated in the same manner. Using the unified notation $\varpi$ it reads:

$$
\varpi^{\theta}=\varpi^{A}+\dot{\varpi}_{i-1} \theta \Delta_{N} t
$$

For the first sub-interval, the stress rate and the hardening parameter rate are initialised through the explicit or forward-Euler scheme.

2. Call the constitutive law to calculate approximated values of the stress and hardening parameter rates:

$$
\begin{gathered}
\tilde{\boldsymbol{\sigma}}_{i}=\boldsymbol{C}\left(\boldsymbol{\sigma}^{\theta}\right)\left(\dot{\varepsilon}-\dot{\varepsilon}^{v p}\left(\sigma^{\theta}, \varpi^{\theta}\right)\right), \\
\dot{\varpi}_{i}=\dot{\varpi}\left(\dot{\varepsilon}^{v p}\left(\boldsymbol{\sigma}^{\theta}, \varpi^{\theta}\right)\right) .
\end{gathered}
$$


3. Calculate new stress and hardening parameter mid-point approximations:

$$
\begin{aligned}
\boldsymbol{\sigma}^{\theta} & =\boldsymbol{\sigma}^{A}+\tilde{\boldsymbol{\sigma}}_{i} \theta \Delta_{N} t, \\
\varpi^{\theta} & =\varpi^{A}+\dot{\varpi}_{i} \theta \Delta_{N} t
\end{aligned}
$$

4. Repeat 2. and 3. untill it converges. It is supposed that the convergence is obtained after two iterations.

5. Calculate the stress state at the end of the sub-interval:

$$
\boldsymbol{\sigma}^{B}=\boldsymbol{\sigma}^{A}+\tilde{\boldsymbol{\sigma}}_{i} \theta \Delta_{N} t
$$

and update the hardening function:

$$
\varpi^{B}=\varpi^{A}+\dot{\varpi}_{i} \theta \Delta_{N} t
$$

6. Take into account an Jaumann correction based on the mid-point stress value:

$$
\boldsymbol{\sigma}^{B}=\boldsymbol{\sigma}^{B}+\boldsymbol{\omega} \sigma^{\theta}+\boldsymbol{\sigma}^{\theta} \boldsymbol{\omega}^{T}
$$

The above described updating procedure depends on the current value of the stress invariant $I_{\sigma}$, which dictates the type of the activated deformation mechanism. If $I_{\sigma}<L$ then cap constitutive equations (5)-(10) are employed in 2. and for $I_{\sigma} \geq L$ equations (11)-(19) are used.

\section{Numerical Example}

Finite element simulation of one-dimensional consolidation has been performed. Plain strain state has been considered. The material property data is: mass density of solid skeleton $\rho=2.647 \mathrm{kN} \mathrm{s}^{2} / \mathrm{m}^{4}$ and the initial porosity $n=0.332$. Elastic properties are caracterised by constants: $E=3.6 \times 10^{5} k P a, \nu=0.3$. For the viscoplastic response material constants are: $\phi_{C f}=\phi_{C 0}=32^{\circ}, \phi_{E f}=$ $\phi_{E 0}=52^{\circ}, c_{f}=c_{0}=10 \mathrm{kPa}, B_{p}=0.0001, B_{c}=0.0002, n=-0.229, \alpha_{c}=0.9$, $\alpha_{d}=0.1, \omega=2.0 \times 10^{-5}, a_{2}=1.3, \iota=0.52$ and the reference stress $p_{a}=$ $1.0 \times 10^{8} \mathrm{kPa}$. The sizes of the sample are $3.00 \times 3.00$ meters. Finite element mesh and boundary conditions are shown on Fig.1, $a$. For the numerical simulation a multistage rapid loading path followed by creep has been applied such that for $0 \leq t \leq 4 \mathrm{~s}$ there is loading up to $1.2 \mathrm{MPa}$, for $4 s<t \leq 236 \mathrm{~s}$ the load is kept constant, for $236 \mathrm{~s}<t \leq 240 \mathrm{~s}$ loading up to $2.4 \mathrm{MPa}$ and for $t>240 \mathrm{~s}$ there is a creep with a constant load of $2.4 M P a$. Fig.1, $b$. illustrates a typical variation of the vertical displacement with the loading history at nodal points 7 and 9 .

\section{Conclusions}

The viscoplastic formulation and the numerical algorithm presented provide a general format for extending inviscid models to Perzyna-type viscoplasticic constitutive relationships suitable for finite element applications. The problem of 


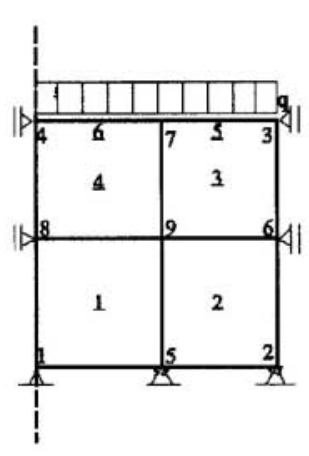

a.

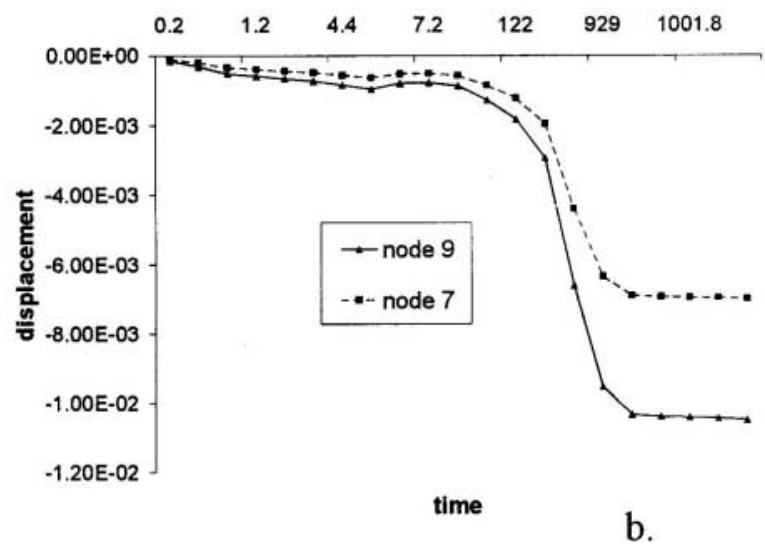

b.

Fig. 1. a. Finite element mesh and boundary conditions for the numerical example. b. Displacement versus time in nodal points 7 and 9 - a representative result

Perzyna's viscoplasticity extension to multisurface inelasticity is solved by dividing the stress space on subspaces depending on the value of the first stress invariant and by defining for each subspace loading and potential functions properly modelling the deformatiom mechanism activated by stress states belonging to the given subspace. As an application of this concept, a viscoplastic model for high porous rocks capable of describing experimentally observed shear failure and collapse deformation mechanisms is presented. The model is implemented into the Lagamine finite element code. A numerical test example for solving one-dimentional nonlinear consolidation shows a reasonable prediction, qualitatively representing the experimental observations during oedometer creep tests on chalk, [8]. The experience with the Lagamine FE code shows that the selection of the time step length is very important for the accuracy of the solution. The variable time stepping scheme, realized by Lagamine automatic strategy is more advantageouse to achieve the solution accuracy. Further work is needed for evaluating proper identification techniques and experimental verification of both elastic and inelastic behaviour. The experimental data has to be suffitiant for performing a more precise least - square nonlinear estimation procedure. Thus it will be possible to compare not only qualitatively but also quantitavely the experimental and numerical results.

\section{Acknowledgements}

The NATO Science Committee is gratefully acknowledged for supporting the first author by Type B (Advanced) Fellowship to work in DIG, University of Liege, Belgium. Partially this work has been sponsored by the National Science Fund, Bulgaria within the Research project MM 903/99. 


\section{References}

1. Collin, F., Radu, J-P., Charlier, R.: First development of the mechanical model. PASACHALK - 1st periodic twelve-monthly progress report . (1998) 222

2. Collin, F., Delage, P., Schroeder, C., Charlier, R.: Geomechanical constitutive modelling of a chalk partly saturated by oil and water. EUROCK 2000 Symposium. Aachen, Germany, 27-31 March. (2000) 222

3. Barnichon J.-D.: Finite Element Modelling in Structural and Petroleum Geology. PhD thesis, Université de Liège, (1998) 225

4. Van Eekelen H. A. M.: Isotropic yield surfaces in three dimensions for use in soil mechanics. International Journal for Numerical and Analytical Methods in Geomechanics. No. 4. pp. 98-101 (1980) 225

5. Charlier R.: Approche unifiée de quelques problèmes non linéaires de mécanique des milieux continus par la méthode des éléments finis. Université de Liège. Thèse de Doctorat. (1987) 226

6. Perzyna P.: Fundamental Problems in Viscoplasticity. Advances in Applied Mechanics, vol. 9, 244-368 (1966) 222, 223

7. Simo J. C. and T. J. R. Hughes: Computational Inelasticity. Springer Verlag, Berlin (1998) 224

8. Shao J. F. and Henry J. P.: Development of elastoplastic model for porose rock. International Journal of Plasticity. vol. 7, 1-13, (1991) 223, 228 